\title{
Developing food-based dietary guidelines for 1-5 year old children: a protocol for use in population health globally
}

\author{
$\underline{\text { O.C. Lyons }}^{1,2}$, H. McNulty ${ }^{1}$, M.A. Kerr ${ }^{1}$, F. Ward ${ }^{3}$, M.B.E. Livingstone ${ }^{1}$, I. Saul ${ }^{4}$ and \\ M.A.T. Flynn ${ }^{2,1}$ \\ ${ }^{1}$ Ulster University, Coleraine, United Kingdom, \\ ${ }^{2}$ Food Safety Authority of Ireland, Dublin, Ireland, \\ ${ }^{3}$ Our Lady's Children's Hospital, Dublin, Ireland and \\ ${ }^{4}$ Scientific Committee of the Food Safety Authority of Ireland, Dublin, Ireland
}

\begin{abstract}
Early childhood is a well-established critical period for growth and development, potentially impacting on life-long health. Healthy dietary habits formed during the transition from a predominantly milk-based to a food-based diet track into later life. Globally, there is no established process for developing food-based dietary guidelines (FBDG) for 1-5 year old children. This study aims to establish a protocol for developing FBDG for 1-5 year old children for use in population health globally.

Foods consumed by $>10 \%$ of consumers aged 1-5 years (at each eating occasion) were identified by secondary analysis of the Irish National Pre-School Nutrition Survey (NPNS; 2012). Consultations were held with registered dietitians to update the NPNS data and reflect current dietary habits. Dietary modelling, based on healthy eating principles, was conducted on boys $(n 30)$ and girls $(n 30)$ at five percentiles on the World Health Organisation (WHO) growth charts $\left(0.4^{\text {th }} ; 25^{\text {th }} ; 50^{\text {th }} ; 75^{\text {th }} ; 99.6^{\text {th }}\right)$ and at six age time-points $(1 \mathrm{y}$; $1.5 \mathrm{y} ; 2 \mathrm{y} ; 3 \mathrm{y} ; 4 \mathrm{y}$ and $5 \mathrm{y})$. Intake targets were identified for energy, macronutrients and 6 key micronutrients. For those with inadequate nutrient intakes, key contributing foods were identified and used in the modelling.

Dietary modelling yielded 640 four-day food intake patterns. For 1-3 year olds, especially those $<25$ th growth percentile, iron was identified as an at-risk nutrient as the intake target was not achieved. For all $1-5$ year olds, vitamin D was identified as an at-risk nutrient. Red meat and iron-fortified cereal $(>12 \mathrm{mg} / 100 \mathrm{~g})$ were identified as key contributors to iron intake. A combination of red meat (30 g, 3 days/week) and iron-fortified cereal (30 g, 5 days/week) resolved inadequate iron intakes for 1-3 year olds, except those $<25$ th growth percentile. For those children, the additional inclusion of $4 \mathrm{mg}$ iron from use of iron-fortified milk $(1.2 \mathrm{mg} / 100 \mathrm{~mL})$ or a low-dose iron supplement $(7 \mathrm{mg}, 4$ days/week) resulted in adequate iron intakes. For all children aged 1-5 years, vitamin D intakes improved by including a daily $5 \mu \mathrm{g}$ vitamin $\mathrm{D}$ supplement, but still did not reach the intake target.

Worldwide, significant resources are invested in assessing growth and development of 1-5 year olds. This study provides a protocol for developing FBDG to meet nutritional needs of 1-5 year olds at various growth parameters (age and percentiles), using WHO charts. This enables the provision of practical food-based interventions to nutritionally vulnerable children. Using national dietary data, this approach can be applied for developing FBDG specific to a country's needs.
\end{abstract}

\section{Conflict of Interest}

There is no conflict of interest 